Preprint LA-UR-97-1634

$$
\text { CONF-970503--235 }
$$

Title: SIMULATIONS OF THE LEDA RFQ 6.7 MEV ACCELERATOR

Author(s): Lloyd M. Young

Submitted To: 1997 Particle Accelerator Conference Vancouver, BC, Canada May 12-16, 1997
LANSCE-1

\section{RECEIVED \\ AUG 131997 \\ OSTi.}

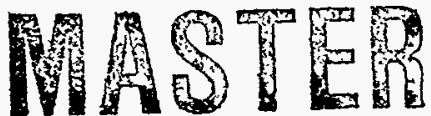

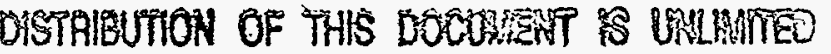

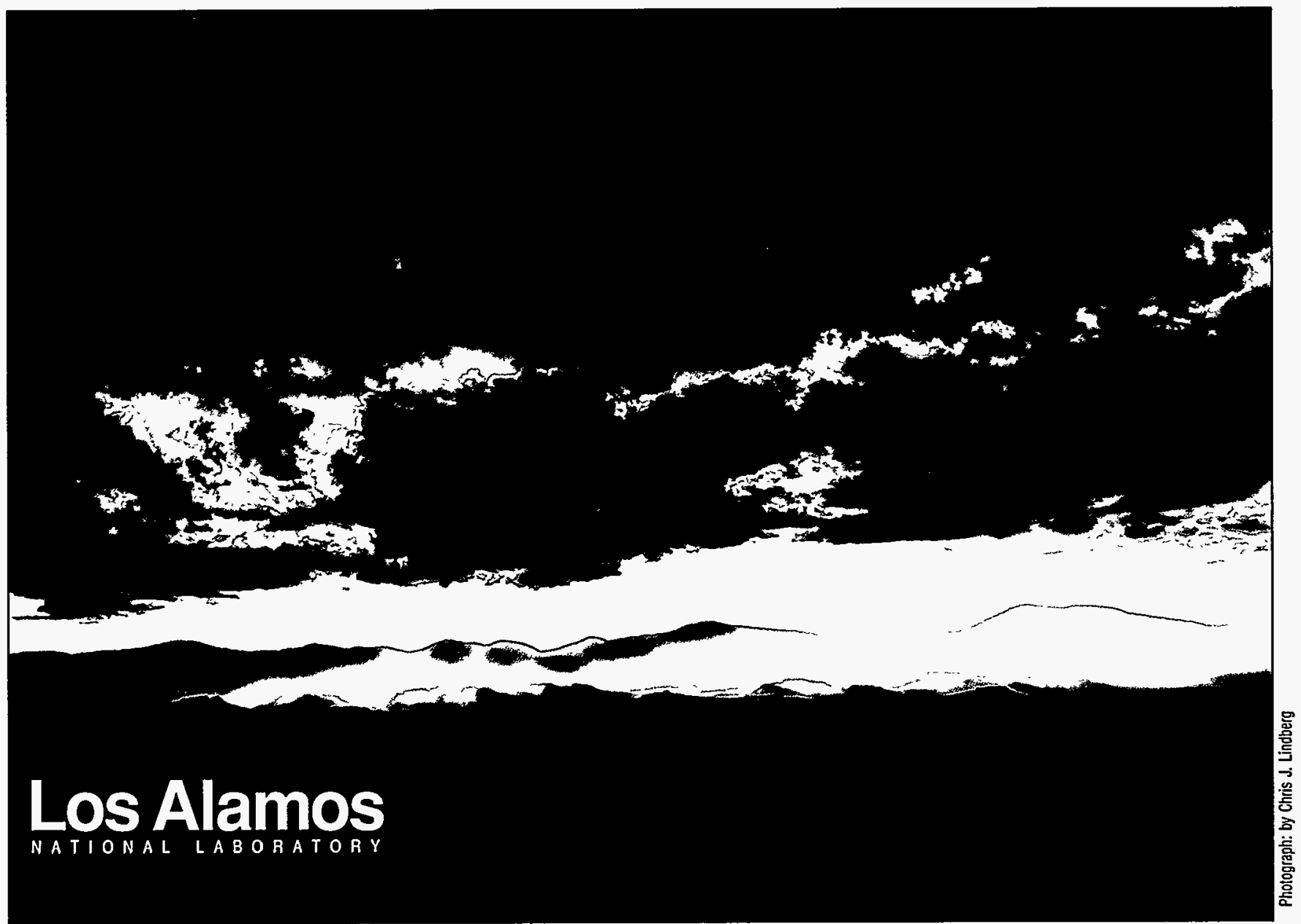

This is a preprint of a paper intended for publication in a journal or proceedings. Because changes may be made before publication, this preprint is made available with the understanding that it will not be cited or reproduced without the permission of the author. 


\title{
SIMULATIONS OF THE LEDA RFQ 6.7-MEV ACCELERATOR *
}

\author{
Lloyd $M$. Young \\ Los Alamos National Laboratory, MS H817, Los Alamos, NM 87545, USA
}

\begin{abstract}
The codes PARMTEQM and RFQTRAK simulate the beam transport through the radio-frequency-quadrupole (RFQ) accelerator for the low-energy-demonstration accelerator (LEDA). They predict $95 \%$ transmission for a matched $110-\mathrm{mA}$ proton beam with a normalized-rms emittance of $0.02 \mathrm{~mm}$ mrad. RFQTRAK simulates the effects of arbitrary vane-tip misalignments. This RFQ includes some new features in its design. It consists of four resonantly coupled 2-m-long segments that make up its 8-m length. It has higher vane-gap voltages at the highenergy end than the low-energy end. The entrance end of the RFQ has lower transverse focusing strength to facilitate beam matching. The exit of the RFQ has a transition cell and a radial-matching section. The exit radial-matching section matches the beam into the following accelerator.
\end{abstract}

\section{INTRODUCTION}

LEDA requires $100 \mathrm{~mA}$ of beam from the $350-\mathrm{MHz} \mathrm{RFQ}$. The energy needs to be as high as possible for injection into the coupled-cavity drift-tube linac (CCDTL). Therefore, the LEDA RFQ is an 8-m-long RFQ that accelerates the proton beam to $6.7 \mathrm{MeV}$. A recent experiment with the LEDA RFQ low-power model[1] demonstrates resonantly coupling four 2 -m-long segments to form an 8-m-long RFQ. The LEDA RFQ will use this configuration.

Conventional RFQ designs with a small entrance aperture require a very strongly focused beam at the entrance aperture for proper matching to the RFQ. In LEDA the final lens in the low-energy-beam transport (LEBT) is far enough from the input of the RFQ to require a large aperture and weak focusing at the beginning of the RFQ. Low vane moduiation at the RFQ entrance allows weaker focusing, but still has a large transverse current limit. The combination of a largeaperture radial matching section and weak focusing makes matching the beam into the RFQ easy. As shown in Fig. 1, the transverse focusing strength smoothly increases in the first $32 \mathrm{~cm}$ of the RFQ. At about $130 \mathrm{~cm}$, the vane gap voltage starts ramping up, the aperture starts increasing, and the focusing starts decreasing. The combination of these parameters reduces the beam loss at the end of the gentle buncher, which is the usual choke point where significant beam loss occurs.

The increase in gap voltage increases the accelerating gradient in the high-energy portion of the RFQ and shortens the length. The transverse focusing at the end of the RFQ matches the transverse focusing of the CCDTL, which makes the transition independent of the beam current.

*Work supported by the US Department of Energy.

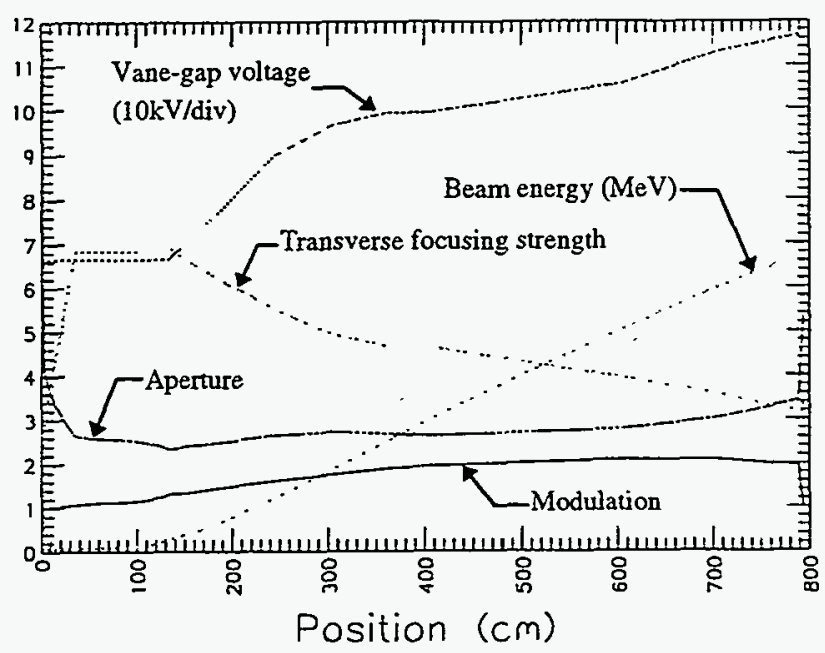

Figure 1. Shows some important parameters versus position in the RFQ.

\section{PROGRAM RFQTRAK}

RFQTRAK [2] tracks the particles through the RFQ using time as the independent variable. Particles enter a cell pair with known phase, position, and divergence angle. For each time step, RFQTRAK advances all the particles and calculates the space-charge-potential distribution. Tracking through the cell pair takes 3 RF cycles. The first cycle, which does not include space-charge fields provides the leading pulse. The third cycle provides a trailing pulse for the correct calculation of the spacecharge fields. At start of the second cycle, RFQTRAK initiates the space-charge calculation. The code discards particles whose velocity falls below half the cell's synchronous velocity. Particles from the second cycle that actually exit the cell pair, provide the starting coordinates for all three cycles in the next cell pair.

RFQTRAK constructs a finite-element model of the potential distribution inside an RFQ accelerator cell. The mesh generator includes details of the topology of an RFQ cell. Mesh elements are three-dimensional bricks with 20 nodes. This number of nodes allows a quadratic variation of the potential in each dimension. The program RFQCOEF [3] calculates the first 8 multipole components of the potential in one quadrant of a single cell or optionally in all 4 quadrants of the cell. PARMTEQM [4] uses the same 8 multipole components. The 4-quadrant calculation also includes the dipole and sextupole terms.

RFQTRAK employs another finite-element mesh similar to the one used for the potential distribution to calculate the effects of space charge during the beam dynamics simulation. In this calculation, the image charge effect is naturally included by the boundary conditions. In this paper, the term space-charge effect includes the image charge effect. The finite-element model spans a pair of cells of total length $\beta \lambda$, where $\beta \lambda$ is the distance traveled 
by a particle of velocity $\beta c$ in one RF period. For solving Poisson's equation: $\nabla^{2} \Phi=-4 \pi \cdot \rho$, the right-hand terms come from the beam charge distribution. The vane potentials are assigned to be zero as a boundary condition. The code maps the potential on nodes of the entrance plane to the corresponding nodes on the exit plane. A direct (Gauss Elimination) method solves for the potentials. An advantage of this method is that the solution matrix can be reused several times, with only an update of the right-hand side as the charge distribution changes. Then only a back substitution is required to solve for the fields. Space-charge fields are obtained from the potentials by differentiation of the element-shape functions.

\section{THE SIMULATIONS}

RFQTRAK simulated the LEDA RFQ with errors in the vane-tip locations and vane-tip potentials. Table 1 lists a set of randomly generated errors used in these simulations. Figure 2 shows the result of the RFQTRAK simulation with these errors. Notice that the transverse beam size shrinks in the first part of the RFQ where the focusing increases. The code RFQPROC, which is part of the PARMTEQM suite of codes, plotted all the beam distribution figures.

Table 1. Voltage and position errors for RFQ vane tips.

\begin{tabular}{|l|l|l|l|}
\hline Vane & $\begin{array}{l}\text { Voltage } \\
\text { error (\%) }\end{array}$ & $\begin{array}{l}\text { Error in Vane- } \\
\text { tip radial } \\
\text { position (mm) }\end{array}$ & $\begin{array}{l}\text { Error in vane- } \\
\text { tip azimuthal } \\
\text { position (mm) }\end{array}$ \\
\hline 1 & -2.124 & -0.0046 & +0.0231 \\
\hline 2 & -0.159 & -0.0066 & -0.022 \\
\hline 3 & +3.033 & +0.0017 & +0.0030 \\
\hline 4 & -0.751 & -0.0414 & +0.040 \\
\hline
\end{tabular}

Figure 3 shows the phase-space projections at the input of the RFQ and at the output of cell 142. The bold-black points in the input-phase-space projections are particles that were lost before reaching cell 143. At this point in the calculation, the lost particles are the outer most particles injected into the RFQ. The input beam for this simulation came from a simulation of the LEBT beam line[5]. The butterfly shape of the transverse phase-space distributions results from the large variation of the RF phase when the particles reach the cell 142-exit plane. The particles' transverse velocity at the cell exit plane depends on the RF phase.

The ellipses in these plots correspond to the rms Twiss parameters of the beam. The area is 4 times the rms emittance. The separatrix, shown in the lower right of Figure 3 , defines the stable region of the phase-energy plane.

Figure 4 shows the phase space plots from the RFQTRAK simulation at cell 430 , the last regular cell of the RFQ. The butterfly shape visible in the cell-142 transverse phase planes does not appear at cell 430 because the phase spread is small. Compare the RFQTRAK simulation with Figure 5, which shows the same phase-space plots from the PARMTEQM simulation. Tables 2 and 3 list the rms-beam properties of the two simulations.
The LEDA RFQ has a transition cell and a radial matching section[6]. The exit radial-matching section matches the beam into the following accelerator. PARMTEQM can simulate the transition cell and exit radial matching section, but RFQTRAK cannot. Cell 430 is the last cell where a comparison between the two codes is possible. A modification of RFQTRAK will add this capability in the near future.

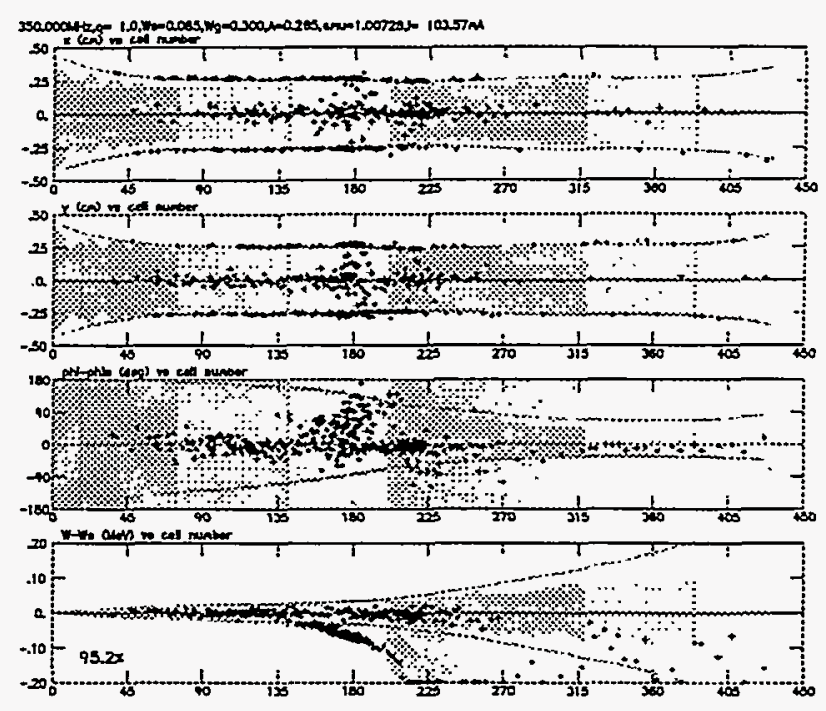

Figure 2. RFQTRAK simulated the LEDA RFQ using 100,000 macro particles. From top to bottom are: $x, y$, phase, and energy coordinates versus cell number. Boldblack points indicate lost particles. Only $10 \%$ of the lost particles are shown for clarity. The percentage transmission is $95.2 \%$.
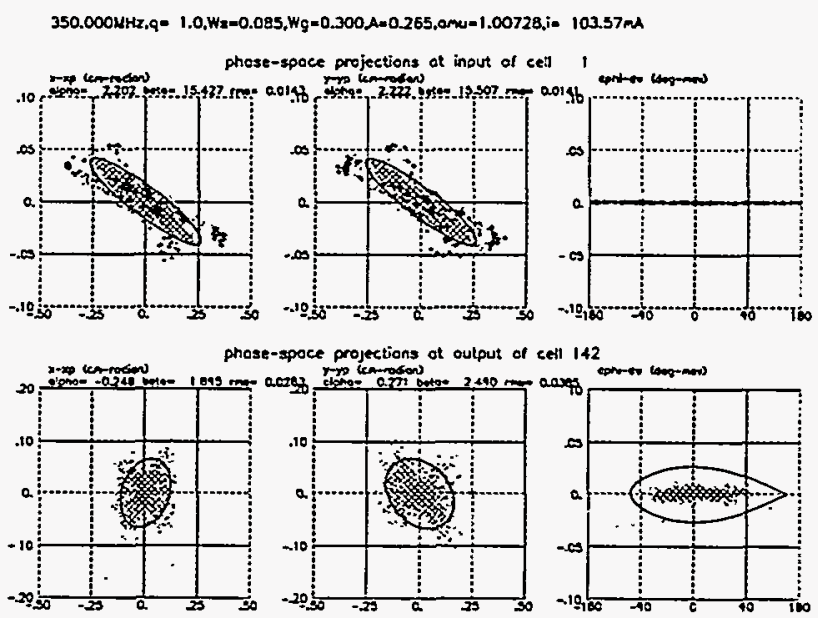

Figure 3. RFQTRAK simulation showing phase-space plots of the input beam and the beam at cell 142 . Only $2 \%$ of the particles are plotted. The bold-black points in the input phase space show $10 \%$ of the particles in the input beam that have been lost up to and including cell 142. The phase-space plots from left to right are: $x-x^{\prime}, y-y^{\prime}$, and phase-energy, where $x^{\prime}=d x / d z$ and $y^{\prime}=d y / d z$.

\section{PARMTEQM}

PARMTEQM, which uses the $z$ position as the independent variable and a cylindrically symmetric space- 


\section{DISCLAMIER}

Portions of this document may be illegible in electronic image products. Images are produced from the best available original document. 


\section{DISCLAIMER}

This report was prepared as an account of work sponsored by an agency of the United States Government. Neither the United States Government nor any agency thereof, nor any of their employees, make any warranty, express or implied, or assumes any legal liability or responsibility for the accuracy, completeness, or usefulness of any information, apparatus, product, or process disclosed, or represents that its use would not infringe privately owned rights. Reference herein to any specific commercial product, process, or service by trade name, trademark, manufacturer, or otherwise does not necessarily constitute or imply its endorsement, recommendation, or favoring by the United States Government or any agency thereof. The views and opinions of authors expressed herein do not necessarily state or reflect those of the United States Government or any agency thereof. 
charge calculation, cannot handle the type of errors listed in Table 1. However, PARMTEQM needs less computer resources and runs several times faster than RFQTRAK. For these reasons, we used PARMTEQM to design the LEDA RFQ. We used the PARMTEQM beam distributions to match the beam into the CCDTL. The validity of the PARMTEQM simulations can be accessed by comparing the beam distributions from PARMTEQM to the distributions from the fully $3 D$ simulation performed in RFQTRAK.
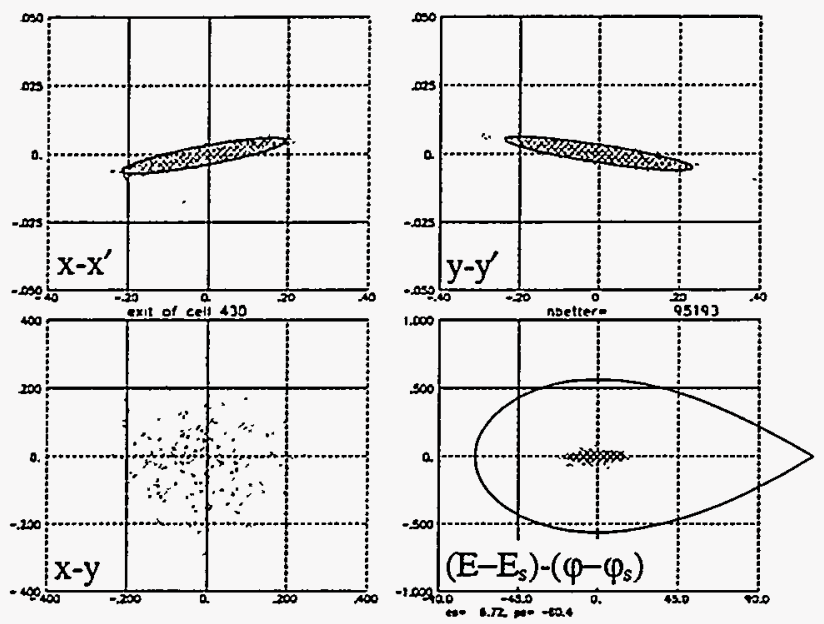

Figure 4 Phase-space projections near the end of the RFQ. Only $2 \%$ of the particles are plotted. RFQPROC calculates the Twiss parameters, $\alpha$ and $\beta$, and the rms emittance $\varepsilon$ from the 95,193 macro particles remaining in the beam. It then draws the ellipses using $4 \times \varepsilon$ for the area. Table 2 lists these Twiss parameters.

Table 2. RFQTRAK Twiss parameters at cell 430.

\begin{tabular}{|l|l|l|l|}
\hline & \multicolumn{3}{|l|}{ Twiss parameters } \\
\hline Parameter & $\mathrm{x}-\mathrm{x}^{\prime}$ & $\mathrm{y}-\mathrm{y}^{\prime}$ & $\left(\mathrm{E}-\mathrm{E}_{s}\right)-\left(\varphi-\varphi_{s}\right)$ \\
\hline$\alpha$ & -1.525 & 1.509 & 0.065 \\
\hline$\beta$ & 59.02 & 73.48 & 424.4 \\
\hline$\varepsilon(\mathrm{cm}-\mathrm{mrad})$ & 0.0211 & 0.0223 & 0.03407 \\
\hline
\end{tabular}

Table 3. PARMTEQM Twiss parameters at cell 430.

\begin{tabular}{|l|l|l|l|}
\hline & \multicolumn{3}{|l|}{ Twiss parameters } \\
\hline Parameter & $\mathrm{x}-\mathrm{x}^{\prime}$ & $\mathrm{y}-\mathrm{y}^{\prime}$ & $\left(\mathrm{E}-\mathrm{E}_{s}\right)-\left(\varphi-\varphi_{s}\right)$ \\
\hline$\alpha$ & -0.750 & 0.591 & -0.087 \\
\hline$\beta$ & 63.297 & 81.752 & 410.2 \\
\hline$\varepsilon$ (cm-mrad) & 0.0172 & 0.0181 & 0.0387 \\
\hline
\end{tabular}

RFQTRAK calculated $95.2 \%$ transmission, which is nearly the same as $95.4 \%$ from PARMTEQM. The most significant difference between beam distributions calculated by the two codes is the difference in the emittance. In RFQTRAK the longitudinal emittance is smaller and the transverse emittance is larger. There is also a difference in the $\alpha$ 's and $\beta$ 's. These differences are large enough that the match to the CCDTL should be checked. RFQTRAK requires the addition of the transition cell and exit radial-matching section before we can make this check. Then the PARMILA code can transport the RFQTRAK particle distribution through the CCDTL. The results of this simulation will provide the required check of the match to the CCDTL.
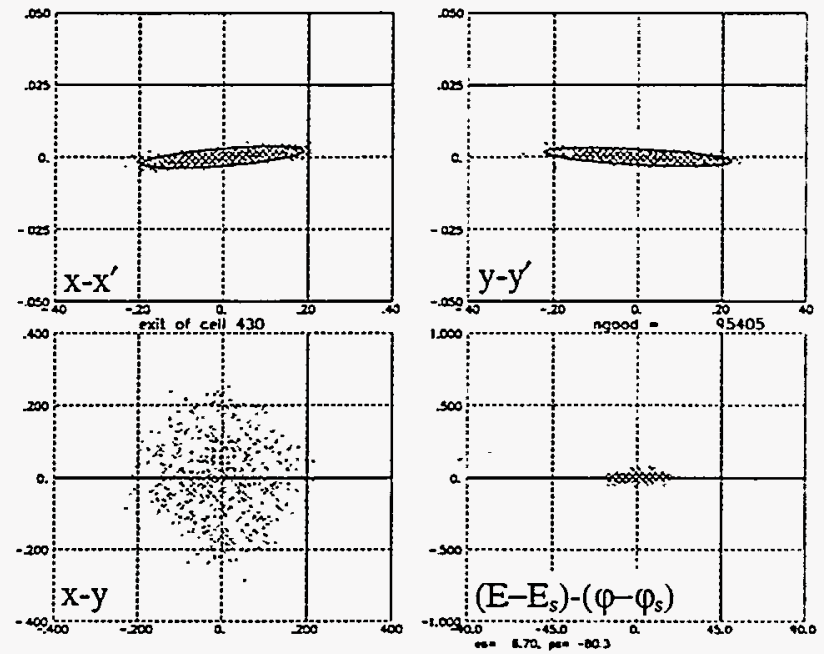

Figure 5. Phase-space projections near the end of the RFQ. Only $2 \%$ of the particles are plotted. PARMTEQM calculates the Twiss parameters, $\alpha$ and $\beta$, and the rms emittance $\varepsilon$ from the 95,405 macro particles remaining in the beam. It then draw the ellipses using $4 \times \varepsilon$ for the area. Table 3 lists these Twiss parameters.

\section{CONCLUSIONS}

RFQTRAK and PARMTEQM simulations are in substantial agreement. The transmission the two codes calculate, with the same input beam, is nearly identical. There are small differences in the emittance and Twiss parameters calculated by the two codes. RFQTRAK needs further development to add the transition cell and exit radial matching section. Then PARMILA could use RFQTRAK's output beam for the CCDTL simulation.

\section{REFERENCES}

[1] L. M. Young, "An 8-meter-long coupled cavity RFQ Linac," Proc. 1994 Intl. LINAC Conf., p. 178 (Tsukuba, Japan, Aug. 21-26, 1994).

[2] N. J. Diserens, "Progress in the Development of a 3D finite Element Computer Program to Calculate Space and Image Charge Effects in RF Quadrupoles," IEEE Trans. Nucl. Sci., NS-32, (5), 2501 (1985).

[3] N. J. Diserens, "RFQCOEF a Package for Extracting the Harmonic Coefficients for the Potential Function in an RF Quadrupole," Proc. 1984 Linear Accel. Conf., Darmstadt, Germany, GSI-84-11, 324 (1984).

[4] K. R. Crandall et al., "RFQ Design Codes," Los Alamos National Laboratory report LA-UR-96-1836. (revision February 12, 1997)

[5] L. M. Young, "Simulations of the LEDA LEBT with $\mathrm{H}^{+}, \mathrm{H}_{2}^{+}$, and $e^{-}$particles," this conference.

[6] K. R. Crandall, "Ending RFQ Vane tips with Quadrupole Symmetry," Proc. 1994 Intl. LINAC Conf., p. 227(Tsukuba, Japan, Aug. 21-26, 1994). 